\title{
Ofatumumab maintenance prolongs progression- free survival in relapsed chronic lymphocytic leukemia: final analysis of the PROLONG study
}

Marinus van Oers ${ }^{1}$, Lukas Smolej ${ }^{2}$, Mario Petrini ${ }^{3}$, Fritz Offner ${ }^{4}$, Sebastian Grosicki $\mathbb{0}^{5}$, Mark-David Levin ${ }^{6}$, Jaclyn Davis ${ }^{7}$, Hiya Banerjee ${ }^{7}$, Tommaso Stefanelli ${ }^{8}$, Petra Hoever ${ }^{8}$ and Christian Geisler ${ }^{9}$

\begin{abstract}
We report the final analysis of the PROLONG study on ofatumumab maintenance in relapsed chronic lymphocytic leukemia (CLL). In all, 480 patients with CLL in complete or partial remission after second- or third-line treatment were randomized 1:1 to ofatumumab (300 mg first week, followed by $1000 \mathrm{mg}$ every 8 weeks for up to 2 years) or observation. Median follow-up duration was 40.9 months. Median progression-free survival was 34.2 and 16.9 months for ofatumumab and observation arms, respectively, (hazard ratio, 0.55 [95\% confidence interval, 0.43-0.70]; $P<0.0001$ ). Median time to next treatment for ofatumumab and observation arms, respectively, was 37.4 and 27.6 months (0.72 $[0.57-0.91] ; P=0.0044)$. Overall survival was similar in both arms; median was not reached (0.99 [0.72-1.37]). Grade $\geq 3$ adverse events occurred in $62 \%$ and $51 \%$ of patients in ofatumumab and observation arms, respectively, the most common being neutropenia ( $23 \%$ and $10 \%)$, pneumonia (13\% and $12 \%$ ) and febrile neutropenia (6\% and $4 \%$ ). Up to 60 days after the last treatment, four deaths were reported in the ofatumumab arm versus six in the observation arm, none considered related to ofatumumab. Ofatumumab maintenance significantly prolonged progression-free survival in patients with relapsed CLL and was well tolerated.
\end{abstract}

\section{Introduction}

As of 2018, chronic lymphocytic leukemia (CLL) remains an incurable disease. Therefore, prolonged progression-free survival (PFS) and overall survival (OS) with good quality of life remain the most important treatment goals. Improvement in PFS can result from more effective induction treatment, and further prolongation may be obtained by maintenance therapy. In 2015, we published results of a planned interim analysis of PROLONG, an open-label, randomized, phase III study that evaluated ofatumumab, a human type I CD20 monoclonal antibody $(\mathrm{mAb})$, as maintenance treatment in patients with CLL who are in at least partial remission (PR) after induction treatment for relapse. Major

Correspondence: Marinus van Oers (m.h.vanoers@amc.uva.nl)

${ }^{1}$ Academisch Medisch Centrum and HOVON, Amsterdam, The Netherlands

University Hospital and Faculty of Medicine, Hradec Kralove, Czech Republic

Full list of author information is available at the end of the article conclusions were that ofatumumab maintenance compared with observation resulted in significant improvement in PFS and was well tolerated ${ }^{1}$. Here we report the final analysis results for key primary and secondary endpoints of the study. Final analysis was triggered by protocol-defined 280 investigator-assessed PFS events.

\section{Materials and methods}

Study design and patients

PROLONG (ClinicalTrials.gov identifier: NCT01039 376) was an open-label, randomized, phase III study conducted at 130 centers in 24 countries in patients with CLL in complete remission (CR) or PR (according to the International Workshop on CLL [IWCLL] updated National Cancer Institute-Working Group [NCI-WG] guidelines $^{2}$ ) after second- or third-line treatment. The inclusion and exclusion criteria have been previously described ${ }^{1}$. The study was conducted according to the 
Declaration of Helsinki and Good Clinical Practice guidelines and approved by the institutional review boards of all participating institutions. Written informed consent has been obtained.

\section{Randomization and treatment}

Randomization (1:1) to ofatumumab or observation was stratified by response at entry (CR or PR), number of previous induction treatments (2 or 3 ) and type of the most recent treatment (chemoimmunotherapy, alkylating monotherapy or other treatment). Crossover was not allowed between study arms.

Within 1 week of treatment assignment, patients in the ofatumumab arm started with intravenous $300 \mathrm{mg}$ treatment, followed 1 week later by 1000 mg every 8 weeks for up to 2 years. Dose reductions were not allowed; however, interruption or delay of administration because of adverse events (AEs) was permitted. Patients were treated until disease progression, withdrawal from study treatment due to unacceptable AEs, consent withdrawal or other reasons. Patients who prematurely discontinued study treatment were included in the analysis regardless of treatment duration.

\section{Assessment of efficacy and safety}

Study parameters assessed at study entry and during follow-up have been previously described ${ }^{1}$. AEs were measured using the National Cancer Institute Common Terminology Criteria for Adverse Events version 4.0.

The primary endpoint was investigator-assessed PFS, defined as the time from randomization to the earliest date of disease progression or death due to any cause. For patients who did not progress or die, PFS was censored at the time of last adequate assessment. In addition, PFS was censored for patients who received new anticancer treatment before disease progression and for those with 2 or more missing assessments. Secondary endpoints included OS, time to next treatment (TTNT), PFS after next-line therapy (defined as the time from randomization until progression or death following next-line therapy) and safety. Responses were defined and assessed according to IWCLL updated NCI-WG guidelines ${ }^{2}$.

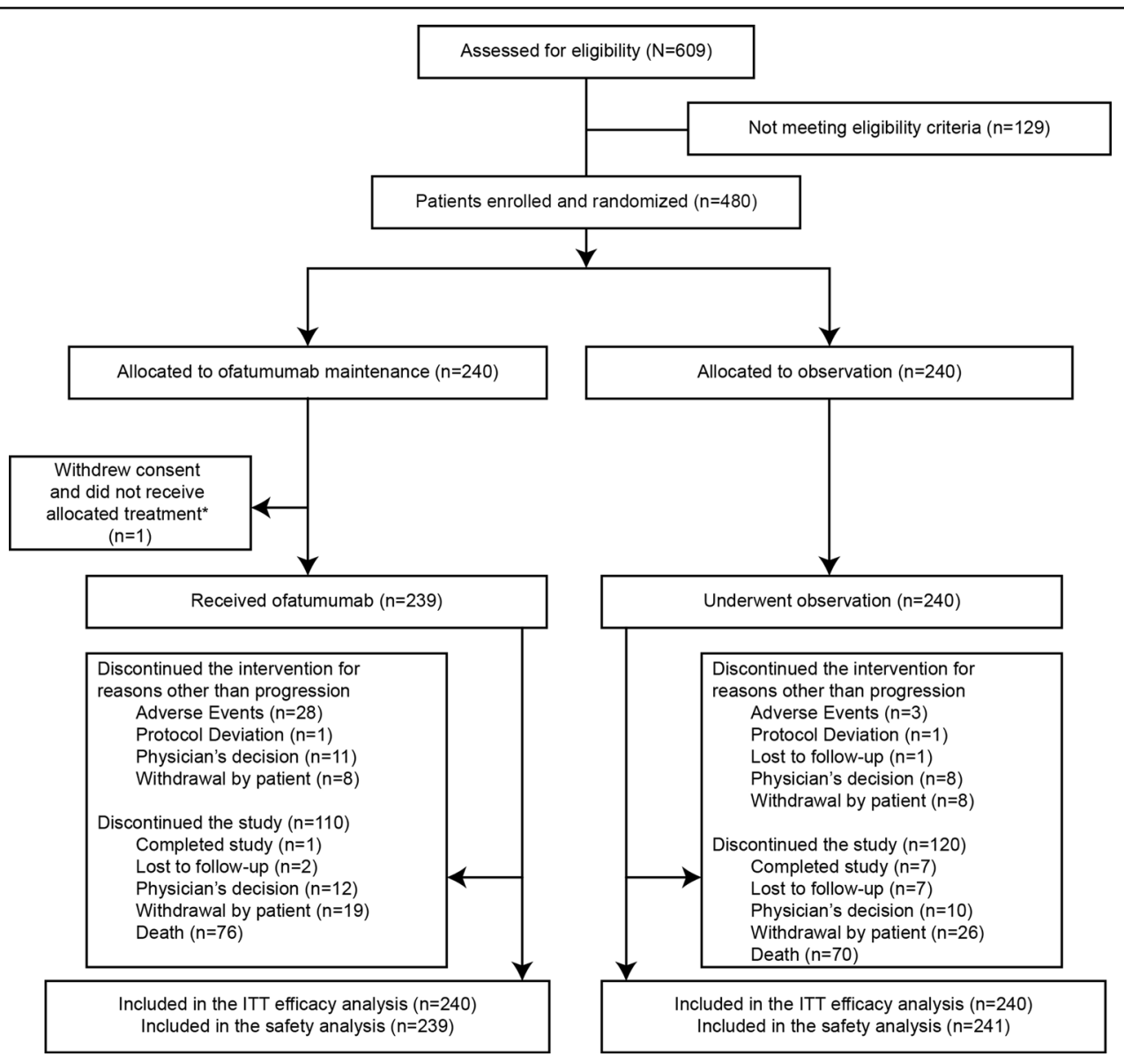

Fig. 1 PROLONG study CONSORT diagram. ITT intent-to-treat. 


\section{Statistical analysis}

Final analysis was conducted when a minimum of 280 events of disease progression or death occurred, which was needed to detect the targeted $40 \%$ improvement in PFS (hazard ratio [HR], 0.71) difference with $80 \%$ power and 5\% two-sided $\alpha$ level. Efficacy analyses were conducted on the intent-to-treat (ITT) population regardless of the actual treatment received. Safety analysis was based on the actual treatment received. PFS, OS, TTNT and PFS after next-line therapy were analyzed using a stratified log-rank test adjusted for stratification factors. Kaplan-Meier curves were generated to determine differences between survival distributions of the treatment arms. All $P$ values are two-sided. Statistical analyses were performed using SAS software (version 9.3; SAS Institute, Cary, NC, USA).

\section{Results}

\section{Patients and treatment}

Between 31 May 2010 and 01 October 2014, 609 patients were screened. A total of 480 patients, including six additional patients enrolled since interim analysis, were randomized to ofatumumab maintenance $(n=240)$ or observation $(n=240)$ (Fig. 1). All 480 patients were included in ITT analyses. One patient from the ofatumumab arm did not receive the allocated intervention (withdrew consent) and was therefore included in the observation arm for safety analysis. At the time of final analysis (cutoff date, 20 February 2017), 146 (30.5\%) patients had died, and disease progression had occurred in 267 (56\%) patients (Fig. 1).

Baseline demographics and clinical characteristics were well balanced between the study arms (Table 1). In total, 385 (80\%) patients were in PR, 94 (20\%) were in CR and 1 $(<1 \%)$ had missing data. Overall, $337(70 \%)$ patients had received 2 prior treatments. The most recent treatment was chemoimmunotherapy in $386(80 \%)$ patients, primarily fludarabine, cyclophosphamide and rituximab (53\%) and bendamustine and rituximab (24\%), while only $23(5 \%)$ patients had received alkylating monotherapy (Table 1); of note, $403(84 \%)$ patients had previously received a rituximab-containing treatment regimen.

Overall, 186 (78\%) patients received $100 \%$ and $42(18 \%)$ received $80 \%$ to $<100 \%$ of the assigned ofatumumab dose. Only $11(5 \%)$ patients received $<80 \%$ of the expected total ofatumumab dose. Primary reasons for ofatumumab discontinuation included AEs (12\%), the most frequent being neutropenia (2\%), refusal/withdrawal by patient (3\%), physician decision (5\%) and protocol deviation $(<1 \%$; Fig. 1$)$.

\section{Efficacy}

At the time of final analysis, the median follow-up duration was 40.9 months. Compared with observation, ofatumumab maintenance resulted in significant and
Table 1 Demographics and baseline disease characteristics ${ }^{\mathrm{a}}$.

\begin{tabular}{|c|c|c|}
\hline & $\begin{array}{l}\text { Ofatumumab } \\
(n=240)\end{array}$ & $\begin{array}{l}\text { Observation } \\
(n=240)\end{array}$ \\
\hline \multicolumn{3}{|l|}{ Age, years ${ }^{\mathrm{b}}$} \\
\hline Median (min-max) & $64.0(33-86)$ & $64.5(39-87)$ \\
\hline$<70, n(\%)$ & $167(70)$ & $166(69)$ \\
\hline$\geq 70, n(\%)$ & $73(30)$ & $74(31)$ \\
\hline$\geq 75, n(\%)$ & $42(18)$ & $35(15)$ \\
\hline \multicolumn{3}{|l|}{ Sex, $n(\%)$} \\
\hline Female & $79(33)$ & $80(33)$ \\
\hline Male & $161(67)$ & $160(67)$ \\
\hline $\begin{array}{l}\text { Time since diagnosis, median } \\
\text { (range) in year }\end{array}$ & $6.0(1-22)$ & $5.0(1-22)$ \\
\hline \multicolumn{3}{|c|}{ Response to last CLL treatment, $n(\%)$} \\
\hline$C R$ & $47(20)$ & $47(20)$ \\
\hline$P R$ & $193(80)$ & $192(80)$ \\
\hline Missing & 0 & $1(<1)$ \\
\hline \multicolumn{3}{|l|}{ Baseline MRD, $n(\%)$} \\
\hline Negative & $31(13)$ & $42(18)$ \\
\hline Positive & $139(58)$ & $107(45)$ \\
\hline Missing & $70(29)$ & $91(38)$ \\
\hline \multicolumn{3}{|l|}{ No. of prior treatments, $n(\%)$} \\
\hline 2 & $169(70)$ & $168(70)$ \\
\hline 3 & $67(28)$ & $63(26)$ \\
\hline Other & $4(2)$ & $9(4)$ \\
\hline \multicolumn{3}{|c|}{ Type of last prior treatment, $n(\%)$} \\
\hline Chemoimmunotherapy & $193(80)$ & $193(80)$ \\
\hline$B R$ & $46(24)$ & $48(25)$ \\
\hline FCR & $100(52)$ & $105(54)$ \\
\hline FR & $4(2)$ & $5(3)$ \\
\hline Other & $30(16)$ & $24(12)$ \\
\hline RCVP & $13(7)$ & $11(6)$ \\
\hline Alkylating monotherapy & $14(6)$ & $9(4)$ \\
\hline Other & $33(14)$ & $38(16)$ \\
\hline \multicolumn{3}{|l|}{ Baseline cytogenetics, $n(\%)^{c}$} \\
\hline 11q deletion & $15(6)$ & $13(5)$ \\
\hline 17p deletion & $7(3)$ & $4(2)$ \\
\hline $\begin{array}{l}6 q \text { deletion or } 12 q \text { trisomy } \\
\text { or } 13 q \text { deletion }\end{array}$ & $47(20)$ & $16(7)$ \\
\hline No aberration & $151(63)$ & $174(73)$ \\
\hline Missing & $20(8)$ & $33(14)$ \\
\hline
\end{tabular}


Table 1 continued

\begin{tabular}{lcc}
\hline & $\begin{array}{l}\text { Ofatumumab } \\
(\boldsymbol{n}=\mathbf{2 4 0})\end{array}$ & $\begin{array}{l}\text { Observation } \\
(\boldsymbol{n}=\mathbf{2 4 0})\end{array}$ \\
\hline IGVH mutational status, $n(\%)$ & $54(23)$ & $74(31)$ \\
Mutated & $139(58)$ & $116(48)$ \\
Unmutated & $3(1)$ & $1(<1)$ \\
Not available & $44(18)$ & $49(20)$ \\
Missing &
\end{tabular}

$B R$ bendamustine and rituximab, $C L L$ chronic lymphocytic leukemia, $C R$ complete remission, $F C R$ fludarabine, cyclophosphamide, and rituximab, $F R$ fludarabine and rituximab, IGVH immunoglobulin variable heavy-chain gene, ITT intent-to-treat, $M R D$ minimal residual disease, $P R$ partial remission, $R C V P$ rituximab, cyclophosphamide, vincristine, and prednisone

${ }^{\mathrm{a}}$ ITT population

${ }^{\mathrm{b}}$ Age was calculated from birth date to screening date in years

c $12 \%$ cutoff

clinically relevant improvement in the primary endpoint. Investigator-assessed PFS was 34.2 months (95\% confidence interval $[\mathrm{CI}], 29.7-38.0)$ for the ofatumumab arm versus 16.9 months (95\% CI, 13.0-20.4) for the observation arm (HR, 0.55 [95\% CI, 0.43-0.70]; $P<0.0001$; Fig. 2a). Assessment of PFS by the independent review committee yielded similar results: 33.6 months $(95 \% \mathrm{CI}$, 28.0-37.2) for the ofatumumab arm versus 15.0 months (95\% CI, 11.4-19.0) for the observation arm (HR, 0.57 [95\% CI, 0.46-0.72]; $P<0.0001)$. When events detected by computed tomography scan were also included, the investigator-assessed PFS was shorter (ofatumumab maintenance 27.5 months [ $95 \% \mathrm{CI}, 23.0$ to 30.2 ] versus observation 13.1 months [95\% CI, 11.6-16.9]; HR, 0.64 [95\% CI, 0.52-0.78]; $P<0.0001$ ). The improved PFS did not translate into a difference in OS, and median OS was not reached in both arms (HR, 0.99 [95\% CI, 0.72-1.37]; Fig. 2b). As shown in the forest plots (Fig. 3), the PFS benefit was evident in most subgroups, including baseline demographic characteristics (age and gender), response at study entry, type of prior therapy, baseline minimal residual disease (MRD) (Supplementary Fig. 1) and immunoglobulin variable heavy-chain gene $(I G V H)$ mutation status. A clear exception was patients with $17 \mathrm{p}$ deletion at relapse on study who did not benefit from ofatumumab maintenance treatment. Although patients with $17 \mathrm{p}$ deletion at baseline (i.e., at study entry) seem to benefit from ofatumumab maintenance, this group is very small, precluding robust conclusions. Unfortunately, results of cytogenetic testing performed at the time of original CLL diagnosis were not available.

Subsequent treatment was administered to 133 (55\%) patients in the ofatumumab arm and 155 (65\%) patients in the observation arm. Ofatumumab maintenance improved TTNT compared with observation (37.4 months [95\% CI, 30.6-42.6] versus 27.6 months [95\% CI, 23.5-32.6], respectively; HR, 0.72 [95\% CI, 0.57-0.91]; $P=0.004$; Fig. 4a). PFS after next-line therapy was defined as the time from randomization to the second objective disease progression or death from any cause, whichever occurred first. Patients who did not progress or die after next-line therapy were censored at their last date of contact. PFS after nextline treatment was not different between the two arms (median not reached; HR, 0.88 [95\% CI, 0.49-1.61]; Fig. 4b). Steroids and some antineoplastic agents (i.e., rituximab, cyclophosphamide, chlorambucil) were used slightly more frequently for next treatment in the observation arm. Interestingly, follow-up therapy for the ofatumumab arm versus the observation arm comprised ofatumumab for 1 $(<1 \%)$ versus $15(9 \%)$ re-treated patients, idelalisib for 5 (3\%) versus $11(7 \%)$ patients, ibrutinib for 54 (35\%) versus $48(29 \%)$ patients, acalabrutinib for $2(1.3 \%)$ versus $0(0 \%)$ patients and venetoclax for $4(3 \%)$ versus $3(2 \%)$ patients, respectively. Thus, about $40 \%$ of patients received novel agents as next-line treatment.

\section{Toxicity}

Both the total number of AEs and number of grade $\geq 3$ AEs were higher in the ofatumumab arm than in the observation arm (Table 2). An increased incidence of grade $\geq 3$ neutropenia was observed in the ofatumumab arm compared with the observation arm (23\% [56/239] versus $10 \%$ [24/241]). Prolonged and severe neutropenia, defined as grade 3 or 4 neutropenia occurring during the treatment period and not resolved at least 42 days after the last dosing date, occurred in 11/239 (5\%) patients in the ofatumumab maintenance arm and 4/241 (2\%) patients in the observation arm. The increased incidence of grade $\geq 3$ neutropenia likely contributed to the observed increase in grade $\geq 3$ infections in patients in the ofatumumab (31\% [73/239]) versus observation (25\% [60/ $241])$ arms. The use of growth factor support was reported 58 times among the 239 patients in the ofatumumab maintenance safety population versus 24 times in the 241 patients in the observation arm. At study entry, in both study arms, median immunoglobulin (Ig) A and IgM levels were decreased, whereas IgG levels were just above the lower limit (Supplementary Fig. 2). During the treatment phase, IgM, IgG, and IgA levels did not change significantly in the ofatumumab arm. In contrast, in the observation arm, serum IgM levels gradually increased to just above the lower limit of normal, whereas IgG and IgA levels did not change. During follow-up, IgM levels showed a slight increase in the ofatumumab arm. Unfortunately, beyond 30 months of follow-up, these small values preclude robust conclusions. Peripheral blood B cells began recovering 3 months after the end of ofatumumab maintenance (data not shown).

Only $4 \%(9 / 239)$ of patients experienced grade $\geq 3$ infusion-related AEs, which were defined as events 


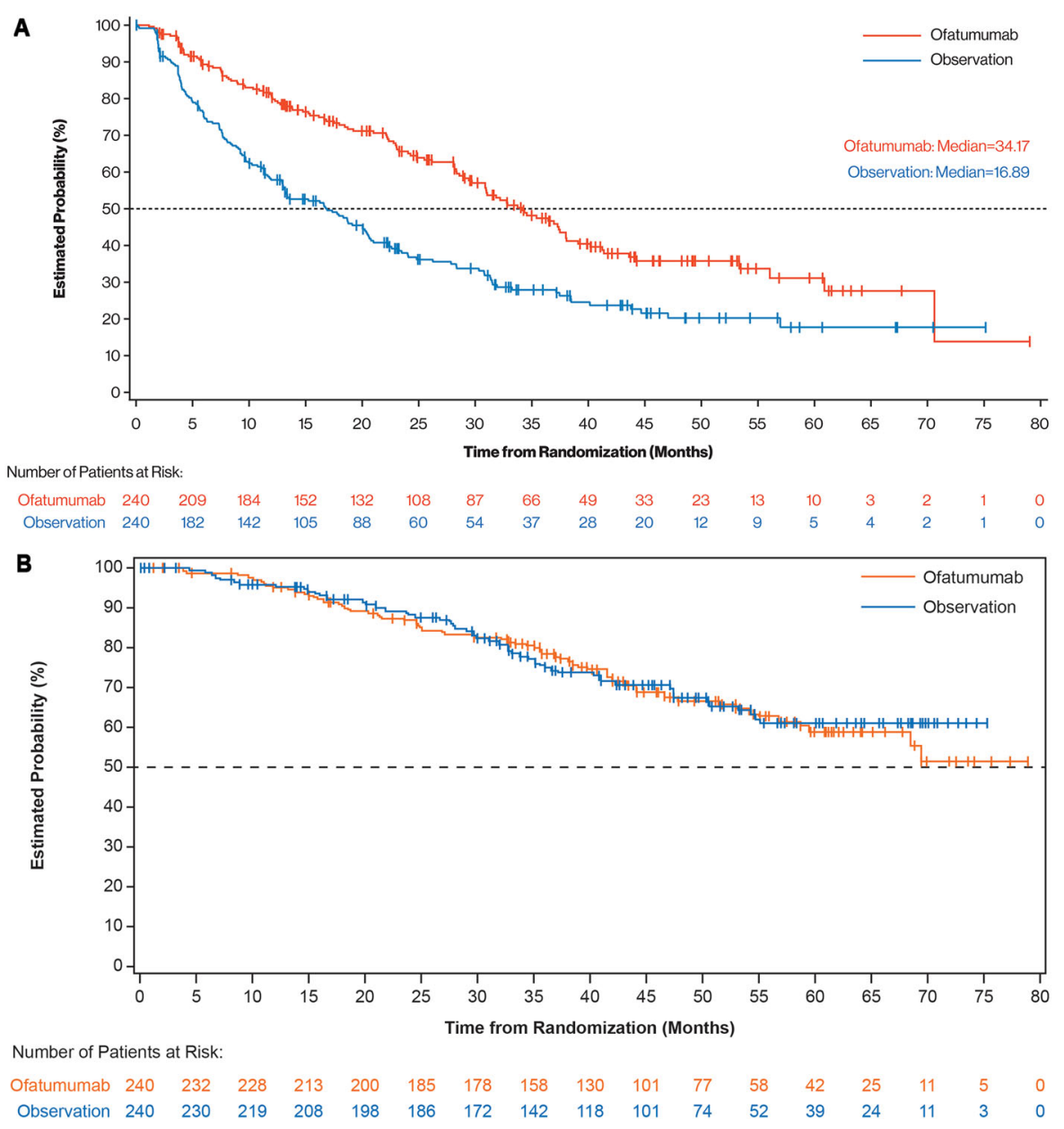

Fig. 2 ITT analysis of a PFS, as assessed by investigator, and $\mathbf{b}$ OS. ITT: intent-to-treat; OS: overall survival; PFS: progression-free survival.

occurring during infusion or within $24 \mathrm{~h}$ after completion of infusion, which the investigator attributed to the treatment medication. These events included, but were not limited to, chills, dyspnea, flushing, hypotension, nausea, pain, pruritus, pyrexia, rash and urticaria. AEs that led to treatment discontinuation occurred in $12 \%$ $(28 / 239)$ of patients in the ofatumumab arm.

During the period from the first dose to 60 days after the last dose, four deaths were reported in the ofatumumab arm (one event each of pneumonia, cerebral haemorrhage, sepsis and small bowel obstruction) and six in the observation arm (two subdural haematoma, one fever and gastric pain, one intestinal infarction, one cardiac arrest, and one disease progression). None of these deaths were attributed to the study drug. At the time of final analysis, a total of 146 (30\%) patients had died: 76 (32\%) in the ofatumumab arm and $70(29 \%)$ in the observation arm. Disease under study was the most frequent cause of death, with 46 (19\%) deaths in the ofatumumab arm and $35(15 \%)$ in the observation arm. Fatal serious AEs occurred in 26 (11\%) patients in the ofatumumab arm and 33 (14\%) patients in the observation arm. Of note, extremely few occurrences of Richter transformation (none in the ofatumumab arm versus 2 in the observation arm) were observed.

\section{Discussion}

Final analysis of the PROLONG study confirmed the interim analysis results ${ }^{1}$ and showed that ofatumumab maintenance improved both PFS and TTNT in patients with relapsed CLL and was well tolerated. The PFS benefit was independent of baseline demographic characteristics, remission status at study entry, prior treatments and $I G V H$ mutation status. Ofatumumab maintenance was accompanied by an increased risk of grade $3 / 4$ neutropenia and grade $3 / 4$ infections. Importantly, ofatumumab maintenance did not increase the risk of Richter transformation nor did it impair the response to next-line treatment.

Although the values are relatively small and our study was not powered to compare these subgroups, the benefit of ofatumumab seems more pronounced in the MRDnegative subgroup (Supplementary Fig. 1). This is an important finding because it shows that patients with 


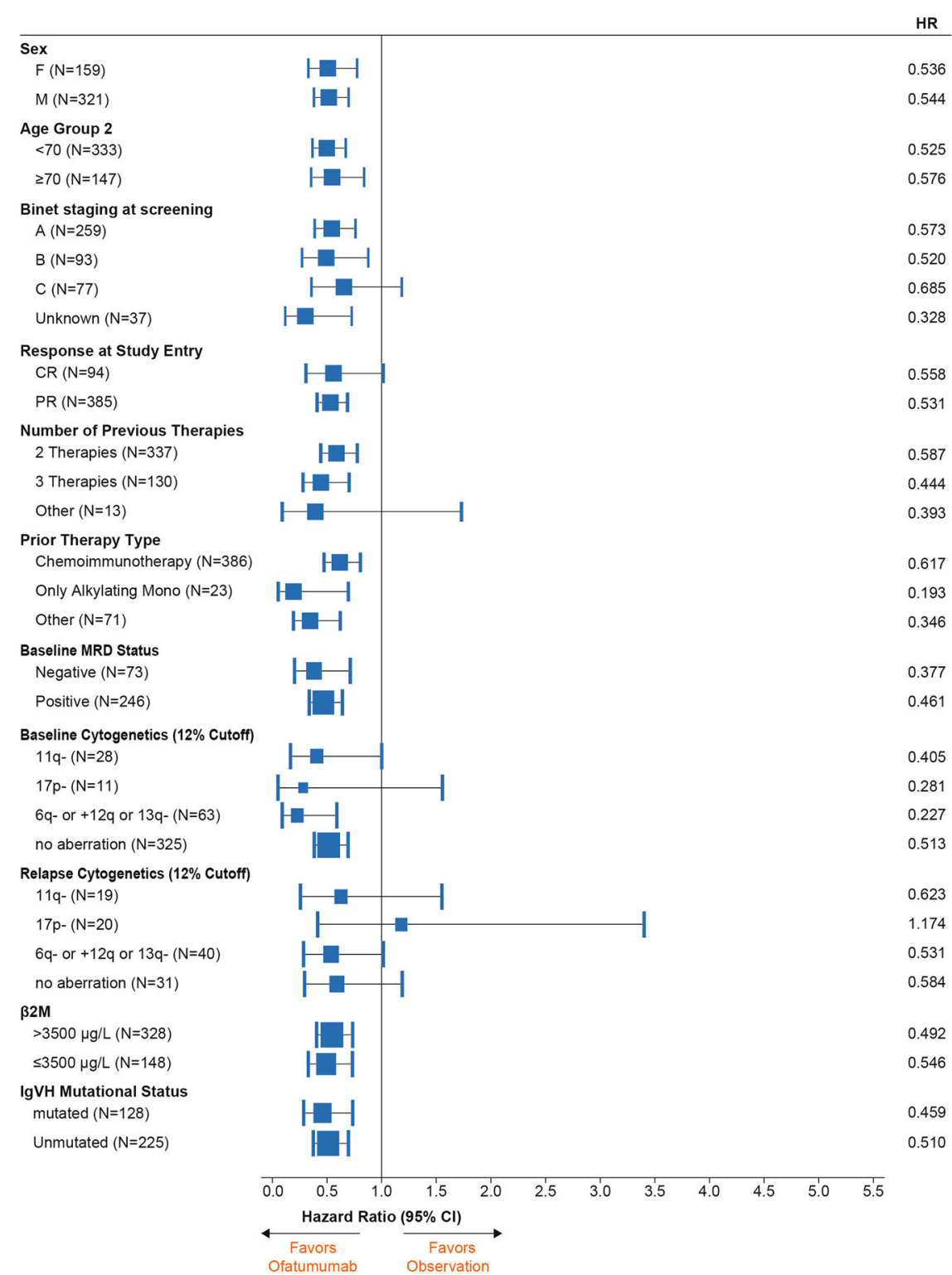

Fig. 3 Forest plot of subgroup analysis of PFS. $\beta 2 \mathrm{M} \beta_{2}$-microglobulin; $\mathrm{Cl}$ confidence interval; $\mathrm{CR}$ complete remission; HR hazard ratio; IGVH immunoglobulin variable heavy-chain gene; MRD minimal residual disease; PFS progression-free survival; PR partial remission.

good response to induction therapy may benefit from additional maintenance therapy. Greil et al. did not find any significant improvement in PFS with rituximab maintenance in patients with CLL who were MRD negative after first- or second-line treatment ${ }^{3}$. Results of long-term follow-up in their lesser pre-treated patient group would certainly be of interest.

Analyses of the cytogenetic subgroups (Fig. 3) should be interpreted with caution because of the very low number of patients with positive testing; this is most likely because patients were in PR or CR at study entry, resulting in levels of cytogenetic abnormalities below the detection threshold, notably in MRD-negative patients. This implies that the group without cytogenetic abnormalities harbors patients with chromosomal aberrations. Unfortunately, data on cytogenetics before study entry were not available. In addition, the low number of patients with $17 p$ deletion might be partially attributable to the fact that these patients probably would not have been considered for participation in our study because they were candidates for other treatment modalities, such as allogeneic stem cell transplantation or treatment with kinase inhibitors. The conclusion that the PFS benefit with ofatumumab maintenance is independent of $I G V H$ mutation status was based on an analysis of a robust number of patients, despite $26 \%$ of patients missing data in both study arms. 

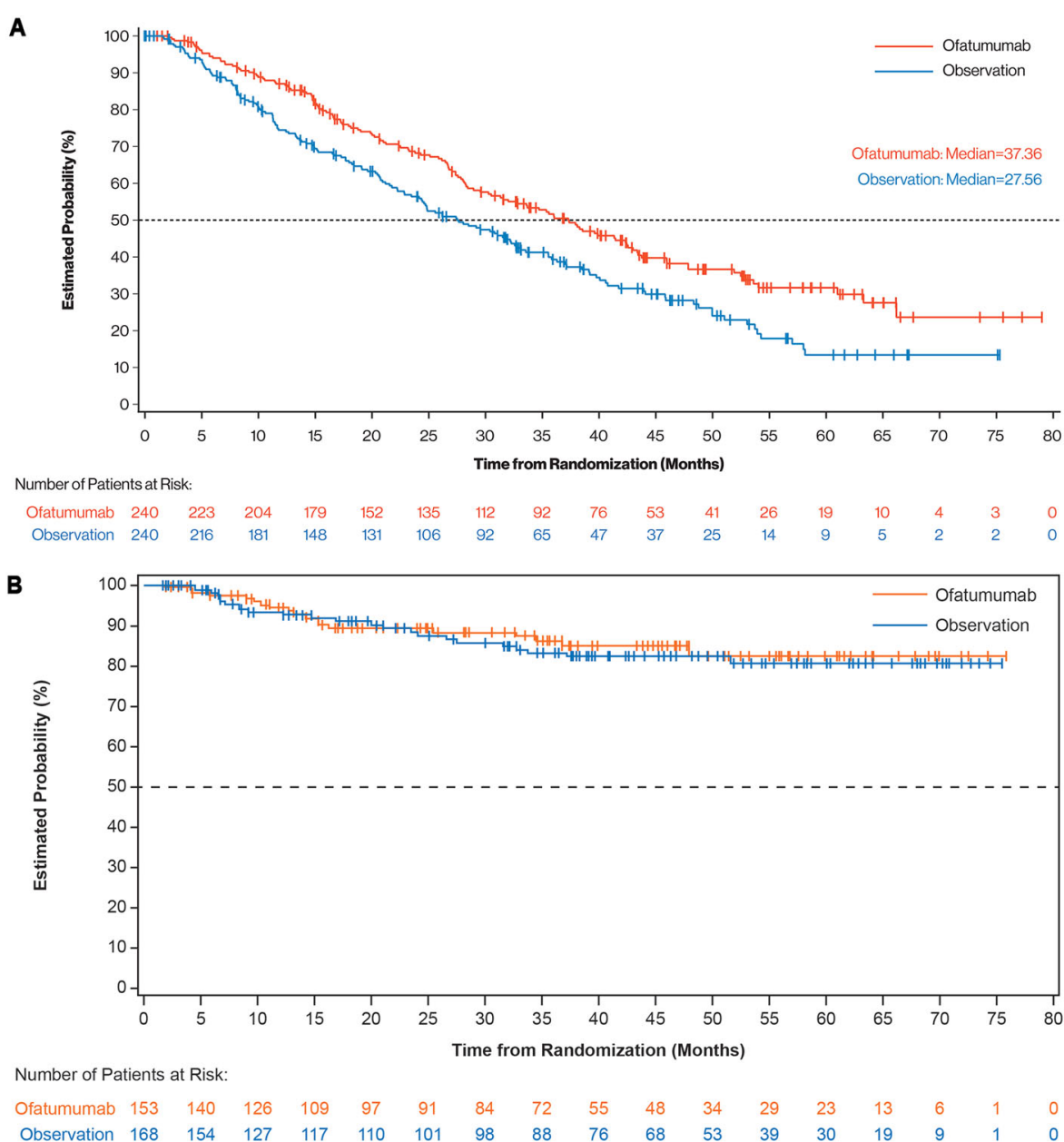

Fig. 4 Time to next-line treatment and PFS after next-line treatment. a TTNT and $\mathbf{b}$ PFS after next-line treatment, defined as the time from randomization to the second disease progression or death from any cause, in patients receiving next-line treatment for relapse in the study. CLL chronic lymphocytic leukemia; PFS progression-free survival; TTNT time to next treatment.

In our study, the interval between progression and next treatment appeared to be longer in the observation arm than in the maintenance arm (Figs. 2a and 4a). More rapid disease progression due to a possible difference in the biology of relapse was not supported by the observed similar PFS after next-line treatment in both study arms (Fig. 4b). Alternatively, lymphocytosis (not necessitating rapid treatment) might be a more frequent mode of progression in the observation arm than in the maintenance arm as long as there is circulating antibody. However, this is not supported by peripheral lymphocyte counts at relapse, which, although slightly lower in the maintenance arm, were not different between the two study arms (Supplementary Table 1). Because CLL progression as such is not an indication to start next treatment, TTNT is susceptible to subjectivity and, therefore, a less robust endpoint compared with PFS. Whether the threshold for initiation of treatment for progression during or after maintenance versus observation was different is speculative. PFS after next-line treatment (consisting of kinase inhibitors in $~ 40 \%$ of patients) in this cohort with 2 or 3 prior treatments is quite remarkable and reflects the progress that has been made in treatment of patients with relapsed CLL.

With longer follow-up, toxicity data were very similar to those found in the interim analysis ${ }^{1}$. Ofatumumab maintenance was associated with an increase in grade $\geq 3$ neutropenia and infections, which was comparable to findings from studies on maintenance therapy with the chimeric anti-CD20 mAb rituximab in both follicular lymphoma ${ }^{4}$ and $\mathrm{CLL}^{3}$. No unexpected toxicity was observed. We have previously shown that no clinically relevant differences in health-related quality of life between the two study arms at any time point during treatment were observed ${ }^{1}$.

At the time of interim analysis in 2014, results of only one randomized phase III trial of maintenance treatment in CLL had been published ${ }^{1}$. In a study of 201 newly diagnosed or previously treated patients with CLL, 
Table 2 Any AEs in $\geq 2 \%$ (grade $\geq 3$ ) of patients by haematologic toxicity and infections.

\begin{tabular}{|c|c|c|c|c|}
\hline \multirow[b]{2}{*}{ Preferred term } & \multicolumn{2}{|l|}{ All Grade } & \multicolumn{2}{|l|}{ Grade $\geq 3$} \\
\hline & Ofatumumab $(n=239)$ & Observation $(n=241)$ & Ofatumumab $(n=239)$ & Observation $(n=241)$ \\
\hline Any event, $n(\%)$ & $209(87)$ & $168(70)$ & $105(44)$ & $74(31)$ \\
\hline \multicolumn{5}{|l|}{ Haematologic toxicity, n (\%) } \\
\hline Neutropenia & $64(27)$ & $27(11)$ & $56(23)$ & $24(10)$ \\
\hline Febrile neutropenia & $17(7)$ & $11(5)$ & $14(6 \%)$ & $9(4 \%)$ \\
\hline Thrombocytopenia & $14(6)$ & $15(6)$ & $5(2)$ & $8(3)$ \\
\hline Anaemia & $9(4)$ & $15(6)$ & $5(2)$ & $7(3)$ \\
\hline Neutrophil count decreased & $8(3)$ & $3(1)$ & $5(2)$ & $2(<1)$ \\
\hline \multicolumn{5}{|l|}{ Infections, $n(\%)$} \\
\hline Pneumonia & $42(18)$ & $41(17)$ & $32(13)$ & $28(12)$ \\
\hline Pyrexia & $51(21)$ & $31(13)$ & $12(5)$ & $6(2)$ \\
\hline Sepsis & 7 (3) & $5(2)$ & 7 (3) & $5(2)$ \\
\hline Septic shock & $5(2)$ & $1(<1)$ & $5(2)$ & $1(<1)$ \\
\hline Lung infection & $4(2)$ & $4(2)$ & $4(2)$ & $3(1)$ \\
\hline Upper respiratory tract infection & $54(23)$ & $28(12)$ & $4(2)$ & $1(<1)$ \\
\hline Herpes zoster & $17(7)$ & $12(5)$ & $3(1)$ & $4(2)$ \\
\hline Urinary tract infection & $13(5)$ & $12(5)$ & $2(<1)$ & $5(2)$ \\
\hline Cellulitis & $5(2)$ & $5(2)$ & $2(<1)$ & $4(2)$ \\
\hline Respiratory tract infection & $18(8)$ & $18(7)$ & $2(<1)$ & $4(2)$ \\
\hline Infusion-related reaction, $n$ (\%) & $42(18)$ & 0 & $3(1)$ & 0 \\
\hline
\end{tabular}

AEs as reported by the investigator

Infusion-related reactions were defined as events occurring during infusion or within $24 \mathrm{~h}$ after completion of infusion and included chills, dyspnea, flushing, hypotension, nausea, pain, pruritus, pyrexia, rash, and urticaria

$A E$ adverse event

rituximab maintenance for 2 years was found to improve PFS and OS but only in high-risk (11q and 17p) patients ${ }^{5}$. Since then, four other randomized phase III studies have been reported. First, final analysis of a multicenter trial on rituximab maintenance for 2 years in 263 patients with CLL (77\% after first-line and 23\% after second-line treatments) showed a median PFS of 47 months in the rituximab arm versus 35.5 months in the observation arm (HR, 0.50 [95\% CI, 0.33-0.75]; $P=0.0007$ ). Median OS was not reached in either arm. Grade 3 to 4 neutropenia occurred in $21 \%$ of patients in the rituximab arm versus $11 \%$ in the observation arm, with grade $\geq 3$ infections in $20 \%$ versus $10 \%$ of patients, respectively ${ }^{3}$. Second, in a randomized, double-blind, placebo-controlled, phase III trial conducted in 314 previously treated patients with CLL, lenalidomide maintenance given until disease progression improved median PFS from 9.2 months in the observation arm to 33.9 months in the lenalidomide arm (HR, 0.40 [95\% CI, 0.29 to 0.55 ]; $P<0.0001)$ but did not improve OS. The most common grade 3 or 4 AEs were neutropenia (lenalidomide arm [60\%] versus placebo arm [23\%]) and thrombocytopenia (17\% versus $6 \%)^{6}$. Third, a randomized, double-blind, phase III study was performed in 89 highrisk patients with CLL, defined as those having post induction intermediate or high MRD levels combined with an unmutated IGVH gene status or TP53 alterations. Lenalidomide maintenance after first-line therapy administered until disease progression improved the median PFS (13.3 months in the placebo arm versus not reached in the lenalidomide arm; HR, 0.168 [95\% CI, 0.074-0.379]; $P<0.0001$ ) but not OS. Grade $\geq 3$ neutropenia occurred in $60 \%$ of patients in the lenalidomide arm versus $23 \%$ in the control $\mathrm{arm}^{7}$. In a French study, 409 treatment-naïve and fit patients with CLL aged $\geq 65$ years in CR or PR after induction with four cycles of fludarabine, cyclophosphamide and rituximab were randomized to rituximab maintenance or observation. Median PFS in the rituximab arm (59.3 months) was greater compared with the observation arm (49.0 months) (HR, $0.55 ; P=0.0002)$. Neutropenia and grade 3 to 4 infections were more common with rituximab maintenance versus observation (53\% versus 36 and $19 \%$ versus $10 \%$, respectively) ${ }^{8}$. 
Taken together, these six randomized phase III studies, four of which included an anti-CD20 mAb, clearly demonstrate the clinical benefit of maintenance treatment in CLL. In all these studies maintenance treatment was applied after induction treatment with chemoimmunotherapy. One might argue that our data are less relevant in the present era of promising data on the use of novel agents in CLL, notably Bruton's tyrosine kinase inhibitors $^{9,10}$, phosphatidylinositol-3-kinase inhibitors ${ }^{11}$ and BCL-2 antagonists ${ }^{12,13}$. Indeed, depending on the outcome of ongoing trials in previously untreated patients comparing "chemofree" induction regimens consisting of (a combination of) novel agents with induction treatment with chemoimmunotherapy, it is expected that upfront use of chemoimmunotherapy in high-risk CLL patients will decrease. However, at present the possible role of chemoimmunotherapy in patients relapsing after prior use of (multiple) novel agents is unclear. Thus for relapsed patients (the population investigated in our trial) our findings might still be relevant. Importantly, thus far the novel agents are all recommended to be continued until relapse, leading to a very prolonged maintenance therapy, despite the limited availability of robust data on long-term safety, including risk of infections, emergence of mutated resistant clones ${ }^{14,15}$ and Richter transformation ${ }^{16}$. Results of ongoing studies on efficacy of kinase inhibitors and BCL-2 antagonists when used for a more restricted period would be relevant for assessment of optimal use of these drugs. In this setting, head-to-head comparisons of efficacy and safety of (prolonged) maintenance therapy with novel drugs versus anti-CD20 mAbs such as ofatumumab are relevant to determine optimal maintenance strategies for patients with CLL. In such studies, quality of life and cost-effectiveness should be important secondary endpoints.

\section{Acknowledgements}

We thank the patients and investigators in the PROLONG study for their participation. We thank Saurabh Agarwal for editorial assistance. The study was funded by GlaxoSmithKline (GSK) and later by Novartis. GSK provided the study drug and worked with the HOVON and Nordic CLL group in the development of the study design. GSK and Novartis worked with the HOVON and Nordic CLL group for collection and interpretation of data.

\section{Author details \\ 'Academisch Medisch Centrum and HOVON, Amsterdam, The Netherlands.

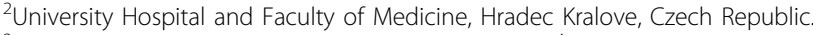 ${ }^{3}$ Azienda Ospedaliero Universitaria Pisana, Pisa, Italy. ${ }^{4}$ Universitair Ziekenhuis Gent, Gent, Belgium. ${ }^{5}$ Department of Hematology and Cancer Prevention, Silesian Medical University, Katowice, Poland. ${ }^{6}$ Albert Schweitzer Ziekenhuis Dordrecht and HOVON, Dordrecht, The Netherlands. ${ }^{7}$ Novartis Oncology, East Hanover, NJ, USA. ${ }^{8}$ Novartis Pharma AG, Basel, Switzerland. ${ }^{9}$ Rigshospitalet- Koebenhavn, Copenhagen, Denmark}

\section{Conflict of interest}

M.V.O. serves as a consulting or advisory role at Roche, ISA Therapeutics and Immunicum and provides expert testimony for Roche. L.S. and M.-D.L. have received honoraria, consulting fees and travel, accommodation and expenses from Roche, Janssen, AbbVie and Gilead. Both also served on speakers' bureau for Gilead. J.D. and P.H. are both employed by and hold stock or other ownership of Novartis. T.S. and H.B. are employees of Novartis. M.P., F.O., S.G., and C.G. have no relationships to disclose.

\section{Publisher's note}

Springer Nature remains neutral with regard to jurisdictional claims in published maps and institutional affiliations.

Supplementary Information accompanies this paper at (https://doi.org/ 10.1038/s41408-019-0260-2).

Received: 10 July 2019 Revised: 18 October 2019 Accepted: 7 November 2019

Published online: 04 December 2019

\section{References}

1. van Oers, M. H. et al. Ofatumumab maintenance versus observation in relapsed chronic lymphocytic leukemia (PROLONG): an open-label, multicenter, randomized phase 3 study. Lancet Oncol. 16, 1370-1379 (2015).

2. Hallek, M. et al. International Workshop on Chronic Lymphocytic Leukemia. Guidelines for the diagnosis and treatment of chronic lymphocytic leukemia: a report from the International Workshop on Chronic Lymphocytic Leukemia updating the National Cancer Institute-Working Group 1996 guidelines. Blood 111, 5446-5456 (2008).

3. Greil, R. et al. Rituximab maintenance versus observation alone in patients with chronic lymphocytic leukemia who respond to first-line or second-line rituximab-containing chemoimmunotherapy: final results of the AGMT CLL-8a Mabtenance randomized trial. Lancet Haematol. 3, e317-e329 (2016).

4. Vidal, L. et al. Rituximab maintenance for the treatment of patients with follicular lymphoma: an updated systematic review and meta-analysis of randomized trials. J. Natl. Cancer Inst. 103, 1799-1806 (2011).

5. Huang, B. T., Zeng, Q. C., Zhao, W. H., Li, B. S. \& Chen, R. L. How to determine post-FCR therapy for cytogenetic risk-tailored elderly patients with chronic lymphocytic leukemia, maintenance rituximab or observation. Med. Oncol. 31, 104 (2014)

6. Chanan-Khan, A. A. et al. Lenalidomide maintenance therapy in previously treated chronic lymphocytic leukemia (CONTINUUM): a randomized, doubleblind, placebo-controlled, phase 3 trial. Lancet Haematol. 4, e534-e543 (2017).

7. Fink, A. M. et al. Lenalidomide maintenance after first-line therapy for high-risk chronic lymphocytic leukemia (CLLM1): final results from a randomized, double-blind, phase 3 study. Lancet Haematol. 4, e475-e486 (2017).

8. Dartigeas, C. et al. Rituximab maintenance versus observation following abbreviated induction with chemoimmunotherapy in elderly patients with previously untreated chronic lymphocytic leukaemia (CLL 2007 SA): an openlabel, randomized phase 3 study. Lancet Haematol. 5, e82-e94 (2018).

9. Byrd, J. C. et al. Targeting BTK with ibrutinib in relapsed chronic lymphocytic leukemia. N. Engl. J. Med. 369, 32-42 (2013).

10. Burger, J. A. et al. Ibrutinib as initial therapy for patients with chronic lymphocytic leukemia. N. Engl. J. Med. 373, 2425-2437 (2015).

11. Furman, R. R. et al. Idelalisib and rituximab in relapsed chronic lymphocytic leukemia. N. Engl. J. Med. 370, 997-1007 (2014).

12. Roberts, A. W. et al. Targeting BCL2 with venetoclax in relapsed chronic lymphocytic leukemia. N. Engl. J. Med. 374, 311-322 (2016).

13. Stilgenbauer, S. et al. Venetoclax in relapsed or refractory chronic lymphocytic leukemia with $17 p$ deletion: a multicenter, open-label, phase 2 study. Lancet Oncol. 17, 768-778 (2016).

14. Komarova, N. L., Burger, J. A. \& Wodarz, D. Evolution of ibrutinib resistance in chronic lymphocytic leukemia (CLL). Proc. Natl Acad. Sci. USA 111 13906-13911 (2014).

15. Woyach, J. A. et al. Resistance mechanisms for the Bruton's tyrosine kinase inhibitor ibrutinib. N. Engl. J. Med. 370, 2286-2294 (2014).

16. Chang, B. Y. et al. Use of tumor genomic profiling to reveal mechanisms of resistance to the BTK inhibitor ibrutinib in chronic lymphocytic leukemia (CLL). J. Clin. Oncol. 31(Suppl. 15), 7014 (2013). 